\title{
Tensiones y desafíos para pensar el cambio en la formación práctica de futuros profesores*
}

\author{
Difficulties and challenges for a change in the practical formation of future teachers
}

Tensões e desafios para pensar a transformação na formaçãoprática de futuros professores

\author{
Carolina Hirmas Ready**
}

\begin{abstract}
RESUMEN
Este estudio aborda los principales tópicos, tensiones y desafíos de la formación práctica en carreras de pedagogía en Chile a partir del análisis de investigaciones y estudios. En la relación teoría-práctica se identifica una tensión importante entre el enfoque conductista-aplicacionista y el crítico-reflexivo, con predominio del primero en el currículum implementado. Bajo el segundo enfoque, las expectativas, creencias y saberes previos de los futuros docentes acerca de la enseñanza y el aprendizaje debieran ser objeto de reflexión, sin embargo a éstos no se les reconoce como sujeto social, situados históricamente, con hábitos y marcos implícitos. Respecto a la triada formativa, existen desacuerdos epistemológicos entre sus actores, lo que dificulta, entre otras cosas, la evaluación de las prácticas. Igualmente, el profesor guía cumple un rol indefinido y solitario dentro de la institución escolar. En suma, se aprecia una relación desarticulada entre las carreras de pedagogía y los centros de práctica que conserva la dicotomía entre teoría y práctica.
\end{abstract}

Palabras clave: desarrollo profesional, práctica, formación docente inicial.

\begin{abstract}
This study addresses the main topics, tensions and oversights from the analysis of research and studies regarding the practical training for teaching careers in Chile. In the theory-practice relationship, an important tension between the behavioral-applicationist and critical-reflective approaches is identified, with predominance of the first curriculum implemented. Under the second approach, expectations, beliefs and prior knowledge of future teachers about teaching and learning should be reflected upon, but apparently it is not recognized as a social subject, historically situated, with habits and implicit frames. Regarding the training triad, epistemological disagreements exist between its members, leading to difficulties in assessment practices, beside other things. The guide teacher plays an indefinite and solitary role within the school. In sum, it prevails a disjointed relationship between teaching careers and practice centers, which preserves the dichotomy between theory and practice.
\end{abstract}

Key words: professional development, practicum, initial training formation.

\section{RESUMO}

Abordam-se os principais tópicos, tensões e desafios, a partir da análise de pesquisas e estudos que dizem respeito à formação prática em carreiras de Pedagogia no Chile. Na relação teoria-prática identifica-se uma tensão importante entre o enfoque condutista-aplicacionista e o crítico-reflexivo, com o predomínio do primeiro no currículo implementado. De acordo com o segundo enfoque, as expectativas, as crenças e saberes prévios dos futuros professores sobre o ensino e a aprendizagem devem ser objeto de reflexão mas, aparentemente, eles não são reconhecidos como sujeitos sociais, situados historicamente, com hábitos e contextos implícitos. Em relação à tríade formativa, há divergências epistemológicas entre seus atores, o que dificulta, entre outras coisas, a avaliação das práticas. O professor orientador

\footnotetext{
Este artículo resume las principales conclusiones del "Estado del Arte sobre formación práctica en carreras de pedagogía", realizado para la OEI Chile el año 2013. Se encuentra actualmente en prensa.

** Con la colaboración de la investigadora Ignacia Cortés
} 
desempenha papel indefinido e solitário dentro da escola. Em suma, nota-se uma relação desarticulada entre as carreiras de Pedagogia e os Centros de prática, conservando a dicotomia entre teoria e prática.

Palavras-chave: desenvolvimento profissional, prática, formação inicial de professores.

\section{INTRODUCCIÓN}

Las prácticas pedagógicas han sido objeto de fuertes cuestionamientos desde el espacio de la academia y desde la observancia ciudadana, dados los magros resultados de desempeño de los estudiantes y el diagnóstico de las deficientes formas de enseñanza observadas en escuelas. Se evalúa que los procesos de formación docente en Chile no han logrado producir los cambios que se requieren para educar y aprender en el siglo XXI (Ávalos y Matus, 2010; Cisternas, 2011; Labra, 2011; Latorre, 2006; Ortúzar et al., 2009). El interés principal de este estudio ha sido presentar y poner en diálogo aquellos temas comunes en los que se enfocan las investigaciones sobre formación práctica de docentes en Chile, identificando coincidencias y divergencias en los objetos de estudio, los enfoques de análisis, principales hallazgos, discusión y conclusiones. Consecuentemente, se identifican tópicos no abordados o insuficientemente tratados por la investigación, cuyas principales conclusiones se resumen en el presente artículo.

Uno de los nudos de interés de la investigación actual refiere al saber docente. Tanto la naturaleza del conocimiento, en sentido amplio, como los procesos a través de los cuales los docentes construyen conocimiento profesional son objeto de estudio (Labra, 2011), en tanto es cada vez más explorado cómo y qué aprenden los docentes (Ávalos y Matus, 2010). Entre los aspectos críticos relevados por los estudios está el de la inadecuada comprensión de la naturaleza teórico-práctica de la pedagogía (Labra, 2011; Latorre, 2006; Montecinos et al., 2010, 2012; Montecinos, Barrios y Tapia, 2011; Montecinos y Walker, 2011; Nocetti et al., 2005; Ortúzar et al., 2009; Rittershaussen et al., 2004; Solís et al., 2011; Walker, 2010). En tal sentido, el carácter situado y distribuido en la construcción del saber docente implica poner gran atención a las interacciones sociales y el contexto en el que éstas tienen lugar (Marcelo, 1999), de modo que el desarrollo del eje de las prácticas docentes cobra especial relevancia dentro del campo de la formación inicial.

A partir del impulso dado por el Programa de Fortalecimiento de la Formación Inicial Docente (PFIDD, 1997-2002), uno de los cambios sustantivos introducidos en los currículos de las carreras fue la incorporación temprana de programas de práctica. Se consideró primordial articular los contenidos teóricos trabajados en la universidad con las experiencias vividas en los centros de práctica. Las críticas más importantes apuntaban a una excesiva teorización que generaba una brecha entre el currículum y los requerimientos del sistema escolar (Ávalos, 2002).

El campo de estudio de la formación práctica es bastante reciente en Chile, aunque ya en el año 1997 Gloria Inostroza realizó una investigación-acción sobre el tema, señalando la experiencia de práctica como motor de la formación docente. Se reconoce su importancia en la formación inicial, como espacio para la reflexión pedagógica y didáctica; para la revisión de creencias sobre la enseñanza y el aprendizaje; para reconocer la influencia de los modelos heredados y aplicados en el ejercicio docente, en contraste con los modelos teóricos o proposicionales (Labra, 2011); así como para la elaboración del saber de la práctica (Galaz y Fuentealba, 2008; Labra, 2011; Latorre, 2006). Por ello, Inostroza propone que el docente debe ser un investigador en el aula. 


\section{METODOLOGÍA DEL ESTUDIO}

Este estudio se basa en el análisis documental de investigaciones y estudios sobre el tema, que presentan cierto nivel de rigurosidad en términos de su congruencia teóricometodológica, su análisis y conclusiones. Los tópicos abordados se desprenden del análisis documental y del diálogo con investigadores en el área. En cada tema se reseñan los estudios que lo tratan, se expone el objeto de estudio, los enfoques, principales hallazgos, discusión y conclusiones. Se analizan 32 publicaciones sobre formación práctica, observándose una creciente actividad investigativa en este ámbito entre los años 1995 y 2013. Sólo en los últimos ocho años (2006-2013) se contabilizan 23 estudios que abordan tanto la práctica profesional como también la intermedia y temprana.

Las investigaciones plantean diversas formas de conceptualizar la práctica en el contexto de la formación inicial de docentes. En Chile, la formación práctica docente se concibe, de manera amplia, como "cualquier actividad que pone al estudiante de pedagogía en contacto con un centro educativo" (Contreras et al., 2010: 92). Así lo resume este equipo de investigadores, tras el análisis de las descripciones del área de formación práctica de las diferentes carreras, considerando las modalidades de trabajo, los propósitos y maneras de establecer el contacto con los centros educativos. La manera tan amplia de definir la actividad de práctica en las carreras impide profundizar en la comprensión sobre sus variados propósitos, contenidos y actividades (Cisternas, 2011). En este sentido, el Proyecto FONDECYT de Montecinos (2010) y Montecinos y Walker (2011) aporta una perspectiva clarificadora, a partir del estudio de 21 carreras de pedagogía básica y media, donde la conceptualización varía en función del nivel de práctica (inicial, intermedia, final) del que se habla.

\section{RESULTADOS}

\subsection{ENFOQUES EN TENSIÓN: CONDUCTISTA-APLICACIONISTA Y CRÍTICO-REFLEXIVO}

La primera interrogante que se plantea este estudio es conocer el sustento conceptual y enfoques epistemológicos de las investigaciones, encontrándose escasos estudios en que se expongan claramente los marcos interpretativos desde los cuales se propone analizar la formación práctica. En este sentido, no es posible encontrar respuestas adecuadas a las preguntas complejas e importantes sobre la preparación de profesores si los estudios adolecen de marcos teóricos comprehensivos acerca de la naturaleza de una adecuada práctica docente y saber profesional.

En aquellos estudios donde se abordan los paradigmas sobre formación docente (Labra, 2011; Molina, 2008; Montenegro y Fuentealba, 2012; Nocetti et al., 2005) se coincide en la tensión de dos enfoques que han regulado la comprensión de la actividad práctica. Un enfoque conductista-aplicacionista o bien técnico-instrumental, en el cual la práctica docente inicial se concibe como una actividad genérica de transmisión de información "desde una perspectiva tradicional sustentada en una racionalidad técnica" (Montenegro y Fuentealba, 2012: s/n). En ésta el practicante asume un rol técnico, pues es el encargado de implementar medidas o "reproducir modelos de enseñanza diseñados por expertos" (Montenegro y Fuentealba, 2012: s/n). Bajo este prisma "la educación [debe] 
cumplir la función de educar para el orden y la cohesión y ayudar a mantener el equilibrio de la sociedad" (Nocetti et al., 2005: 19). La naturaleza del conocimiento es de carácter prescriptivo, se encuentra fragmentado y se desentiende del contexto. En la división dicotómica teoría - práctica, la fundamentación teórica precede a la práctica. Es así que el futuro docente se presenta como un sujeto que es capaz de transmitir conocimientos científicos y culturales (Molina, 2008), predominando un énfasis en el conocimiento de la disciplina (Montenegro y Fuentealba, 2012). Este enfoque sería utilizado por distintas universidades en el mundo para la formación de profesionales (Cohen, 2011 cit. por Labra, 2011; Zeichner, 2010).

El segundo enfoque se plantea como enfoque crítico-reflexivo, se sitúa desde una mirada más comprensiva, reconoce la multidimensionalidad de los fenómenos educativos y la naturaleza situada del conocimiento. La práctica pedagógica, entonces, no debe ser sólo considerada como "un proceso de prueba del proceso de formación" (Labra et al., 2005: 139). Por el contrario, ésta corresponde a un proceso complejo en el que no basta con la transmisión de conocimientos, se trata de una actividad crucial en la formación inicial docente donde los futuros profesores definirán su rol profesional, por lo que debe ser una "una instancia de reflexión y análisis" (Labra et al., 2005: 139). Bajo esta lógica, el saber de la práctica es planteado como una construcción activa por parte del sujeto, basado en la experiencia y el filtro de sus propias matrices de percepción o habitus (Bourdieu, 1987 cit. en Labra, 2011). Se concibe a los profesores como generadores de conocimiento al reflexionar sistemática y colectivamente acerca de su quehacer docente con relación al contexto social e institucional en que se encuentran insertos, y se espera que éstos sean capaces de reconfigurar su saber teórico-práctico.

Con relación a la conceptualización de los actores clave, éstos son identificados como la "triada formativa": estudiante en práctica, profesor supervisor (universidad) y profesor guía (profesor escuela). La forma de denominarlos no suele ser especificada en la mayoría de los estudios, es variable y no incide en la comprensión de sus roles. Esto es importante de definir ya que la manera de denominarlos da cuenta de cómo se les concibe, qué funciones y atribuciones se les otorgan y cómo se entiende el proceso de formación. Para algunos estudiosos en el tema (Gaete, 2003; Montenegro y Fuentealba, 2012), por ejemplo, labor fundamental de ambos formadores es el de articular procesos de investigación-acción grupal (con sus pares futuros docentes), ya que conciben el aprendizaje de la docencia como una práctica investigativa en sí misma, a través del análisis de la racionalidad subyacente en las propias prácticas. Para efectos de este estudio hablaremos del estudiante en práctica o el futuro profesor, resaltando en el primer caso su condición de aprendiz y en el segundo la relevancia que cobra el desarrollo de su rol profesional.

\subsection{PREDOMINIO DE UN ENFOQUE CURRICULAR APLICACIONISTA EN LA RELACIÓN TEORÍA - PRÁCTICA}

A la hora del balance respecto del currículum implementado, los estudios coinciden en el predominio de un enfoque conductista-aplicacionista (técnico-instrumental, mecanicista), en el ejercicio de la práctica, lo que se traduce en entregar información disciplinaria, didáctica y pedagógica que los estudiantes deberán transferir a la docencia. Al parecer, éste es un proceso que caracterizaría en general a la formación inicial docente y que se evidencia en un currículum fragmentado, con escaso trabajo práctico y deficiente 
vinculación con el contexto (Labra, 2011). El peso puesto en la teoría se manifiesta también en el énfasis dado al conocimiento de la disciplina como a la forma de enseñarla (Bobadilla et al., 2009). Esto mismo explicaría que la formación no esté generando las condiciones adecuadas para comprender y enfrentar la complejidad de las situaciones del aula.

El estudio de Montecinos (2010) se adentra en las distintas concepciones sobre la relación teoría-práctica, lo que se relaciona principalmente con el nivel de la práctica. Las prácticas iniciales suelen tener al centro educativo como texto, para que el estudiante observe y haga una lectura de la realidad escolar a partir dela teoría. Las prácticas intermedias se suelen plantear al centro educativo como contexto, como el lugar para ejercitar destrezas y herramientas docentes. En esta fase se pueden dar dos líneas de formación: una que genera reiteradas interacciones con los estudiantes y que incentiva la reflexión docente como una tarea grupal en el uso de herramientas; y otra con escaso contacto con los estudiantes, de carácter prescriptiva. En el primer caso, el futuro docente desarrolla mayor autonomía y una comprensión más compleja de la realidad educativa. La práctica final o profesional se plantea al centro educativo como escenario para poner a prueba las competencias profesionales desarrolladas a lo largo de su proceso de formación. La actividad reflexiva superior, que va de la teoría a la práctica y nuevamente a la teoría, se proyecta como un proceso más intencionado en la práctica final (Montecinos, 2010). Sin embargo, lo que en definitiva se evidencia es que "el avance curricular representa más una acumulación de saberes que una integración de conocimientos en competencias docentes que requieren ser ensayadas, retroalimentadas y reflexionadas de manera interactiva y cíclica a lo largo del proceso formativo, lo que trasunta un remanente de orientación conductista" (Contreras et al., 2010: 100).

Respecto a la progresión en la malla de estudios, los autores (Contreras et al., 2010; Montecinos, 2010; Montecinos et al., 2010; Nocetti et al., 2005; Walker, 2010) coinciden en cuanto a que ésta se organiza de modo que los conocimientos teóricos anteceden al trabajo en el centro educativo. Una tendencia común en la progresión es que ésta va desde la observación a la intervención en competencias aisladas con bajos niveles de autonomía (Walker, 2010), hasta la conducción del proceso de enseñanza y aprendizaje de un grupo curso. En su máxima expresión, el currículo de formación práctica apenas llega a representar una quinta parte de la formación inicial (Walker, 2010). Además, el número de actividades de práctica a lo largo de la formación varía bastante en las distintas carreras, lo que afecta el grado de contacto y complejidad con que es entendida la realidad educativa.

\subsection{VAGUEDAD EN LA IDEA Y CONCRECIÓN DE UN ENFOQUE CRÍTICO-REFLEXIVO}

Desde la perspectiva de un enfoque crítico-reflexivo enunciado en los programas de formación inicial, una definición clave es en qué consiste este ejercicio y sobre qué versa.

Pese a que existe consenso entre los investigadores sobre la importancia de desarrollar reflexión pedagógica, sólo en algunos estudios se define esta idea (Galaz y Fuentealba, 2008; Labra, 2011; Labra et al., 2005; Molina, 2008; Montenegro y Fuentealba, 2012; Nocetti et al., 2005). En la mayoría se plantea de manera muy general sin explicitar niveles o tipos de reflexión a los que se aspira. Los estudios de Labra (2011) y Nocetti et al., (2005) concluyen sobre la escasez de instancias y procesos para desarrollar de manera sistemática habilidades reflexivas, formas de acompañamiento y herramientas cognitivas para activar el diálogo entre teoría y práctica. Labra concluye que el nivel de reflexión alcanzado es 
básico, es decir, los estudiantes en práctica emplean estrategias basadas en un conocimiento técnico y se analizan los resultados a partir de las reacciones de los estudiantes (Leithwood, 1990 cit. por Labra, 2011; Van Mannen, 1991).

Por otra parte, los procesos reflexivos y la incorporación de herramientas de investigación aún no son reconocidos como procesos de profesionalización en el discurso de los actores (triada formativa), como observan Nocetti et al. (2005). Además, los talleres reflexivos están orientados a razonar exclusivamente sobre la acción educativa en los centros escolares y no constituyen una oportunidad de retroalimentación para las universidades o centros de formación docente respecto de su quehacer formativo (Nocetti et al., 2005).

Desde un enfoque crítico-reflexivo, las expectativas, creencias y saberes previos de los futuros docentes acerca de la enseñanza y el aprendizaje debieran ser objeto de reflexión, sin embargo los programas no los consideran, según comentan algunos investigadores (Galaz y Fuentealba, 2008; Labra, 2011). Al parecer, esto se debe a que no se les reconoce como sujeto social, situados históricamente, con hábitos y marcos implícitos.

\subsection{CONTRADICCIÓN EN LOS ESTILOS Y ESTRUCTURAS DE SUPERVISIÓN DESEADOS}

El estilo de supervisión deseable es un tema abordado en algunos estudios. Montecinos, Barrios y Tapia (2011), Solís et al. (2010) y Walker (2010) distinguen entre una orientación reflexiva o directiva en la labor de mediación del supervisor. Frente a actividades más complejas y un modelaje más elaborado o avanzado, los estudiantes en práctica prefieren un estilo de supervisión más reflexivo; frente a actividades más estructuradas optan por un estilo más directivo (Montecinos, Barrios y Tapia, 2011).

Asimismo, el estudio de Walker (2010) concluye que los futuros docentes prefieren un estilo de supervisión más reflexivo junto a grados progresivos de autonomía en la reflexión y toma de decisiones, sin embargo esto se contradice con los temas de conversación que prefieren tener con sus supervisores, los que apuntan a estilos más directivos para el desarrollo de las tareas de práctica. Esta contradicción se explica por un enfoque más instrumental en los modelos de evaluación que socava la orientación más reflexiva que proponen los estudiantes (Walker, 2010). A su vez, Gaete (2003) considera que la dificultad para el diálogo reflexivo y la construcción social del conocimiento estriba en las concepciones autocráticas del conocimiento de los practicantes y sus profesores guías. En este sentido, la propuesta de lineamientos de formación práctica de Montecinos et al. (2010) sugiere, idealmente, un estilo de supervisión flexible de acuerdo a las necesidades de aprendizaje que manifiesta el practicante y que favorezca su creciente autonomía para la toma de decisiones de docencia.

Respecto del saber de la docencia, se constata en algunos estudios que existen desacuerdos epistemológicos entre los actores de la triada formativa. Para Gaete (2003) son precisamente estas concepciones dispares las que dificultan la evaluación del estudiante en práctica. En el estudio de Pardo (2012), mientras los profesores guía comprenden la reflexión desde un paradigma técnico, para el profesor supervisor ésta supone un análisis de orden social y educativo. Tampoco existiría concordancia en la triada sobre los modelos de evaluación (Romero y Maturana, 2012). Los estudiantes en práctica critican la falta de presencia de los profesores supervisores en los centros de práctica (Labra, 2011; Organización de Estados Iberoamericanos [OEI], 2010; Nocetti et al., 2005; Romero y Maturana, 2012). Aparentemente, la ausencia de supervisión se da en mayor medida en 
Enseñanza Media (12\%), respecto a la Educación Básica. En básica, las prácticas inicial e intermedia son guiadas exclusivamente desde la universidad, lo cual no contribuye a cautelar la progresividad en los grados de autonomía (Solís et al., 2011).

Un campo de estudio que requiere mayor investigación refiere a la metodología y prácticas de enseñanza en la formación práctica. De acuerdo con Labra (2011), no se evidencian mecanismos mediante los cuales los profesores supervisores guíen aprendizajes esperados y susciten reflexión sobre lo realizado en contraste con los resultados esperados. Montenegro y Fuentealba (2012) confirman la escasez de estudios referidos a las prácticas de enseñanza y al conocimiento que debiesen poseer los formadores de formadores, y proponen desarrollar al interior de las carreras de pedagogía comunidades de aprendizaje capaces de problematizar acerca de las principales dificultades asociadas al enseñar a enseñar. Desde la perspectiva de los estudiantes en práctica, la aspiración es ser protagonistas en la construcción del conocimiento, demandando crecientes grados de autonomía en las actividades de práctica y en las formas de aprender (Walker, 2010).

Respecto a la evaluación de la práctica, aparte de dos estudios que presentan la validación de instrumentos de evaluación (Abarca, Castaño y Barahona, 2003; Montecinos, 2010) no hay ninguno que investigue qué y cómo se evalúan las prácticas. Gaete (2003); OEI (2010) y Walker, (2010), observan la interferencia de la evaluación en los procesos formativos del futuro docente, puesto que tensionan la relación formativa del profesor guía y/o supervisor con el futuro profesor y contravienen el enfoque crítico reflexivo que se busca intencionar. Para ello se sugiere investigar qué y cómo se evalúa, quién o quiénes evalúan, entre otros aspectos. Los lineamientos curriculares de formación práctica propuestos por Montecinos et al. (2010) proponen evaluar el conocimiento disciplinario, pedagógico y profesional y su uso en el desempeño docente. Los criterios debiesen ser conocidos y compartidos por todos los académicos de la carrera, entregando información válida y confiable respecto de las competencias necesarias para el ejercicio autónomo.

Aparte de la figura del supervisor, no se ha investigado el rol que cumplen otros actores en los procesos de reflexión pedagógica, tales como los compañeros de práctica, coordinadores de práctica y docentes de otras asignaturas de la carrera. Pareciera que entre los investigadores prima un enfoque que concibe el aprendizaje de la docencia como un proceso individual y compartimentalizado en una asignatura: la práctica. Una de las investigadoras destaca la desvinculación entre el área de pedagogía y las áreas de especialidad, respecto a qué y cómo enseñar (Labra, 2011). En este sentido, pareciera que aún no se visibiliza la función primordial en el ejercicio de reflexión pedagógica que pueden cumplir el resto de las asignaturas del currículo de formación inicial en la formación práctica y viceversa. Cómo se articulan las asignaturas de la carrera y el programa de prácticas, es un aspecto no estudiado.

Nocetti et al. (2005) constatan que la estructura de acompañamiento por parte de la universidad se funda en la figura del supervisor, quien es el único encargado de establecer el puente entre la institución de formación docente y el centro de práctica, y el único responsable de monitorear y retroalimentar la actuación de los estudiantes. A este respecto, una idea propuesta en los estudios es la conformación de un equipo docente a cargo de las asignaturas de práctica, el que debiera tener amplio conocimiento del currículo de la carrera y poder ayudar al practicante a "establecer las relaciones pertinentes entre los distintos componentes del currículo que se aplican en el proceso de aprender a enseñar" (Montecinos et al., 2010: 250). 


\subsection{EL PROFESOR GUÍA, ROL INDEFINIDO Y SOLITARIO DENTRO DE LA INSTITUCIÓN ESCOLAR}

Varios estudios confirman la ausencia de mecanismos institucionales de selección de profesores guía adecuados al propósito formativo de futuros docentes (Hass, 2012; Nocetti et al., 2005; Romero y Maturana, 2012). A su vez, un número importante de docentes de aula no tiene suficiente claridad respecto del rol de apoyo que les corresponde cumplir (Montecinos, 2010). En este sentido, no hay una definición al interior de las escuelas de las características de un buen profesor o profesora guía (Nocetti et al., 2005; OEI, 2010; Romero y Maturana, 2012).

Una característica que tensiona la formación del estudiante en práctica es que éste se vincula a la escuela casi en exclusividad a través de la relación con el profesor guía. Además, se evidencia en la escuela un bajo reconocimiento de la experiencia de práctica como experiencia formativa. Existe una mínima interacción del profesor guía con otros miembros de la institución escolar respecto de su función (jefes de UTP, orientadores, inspectores, jefes de ciclo, jefes de departamentos), lo cual dificulta que se construya socialmente su función de guía. En suma, no se ha institucionalizado en la escuela la labor de formación del futuro docente (OEI, 2010).

En cuanto a la disposición de los profesores guías a actuar como formadores de docentes, mientras en el estudio de Montecinos (2010) los estudiantes en práctica declaran en su mayoría percibir el interés de éstos en apoyar sus aprendizajes, según el estudio de Nocetti et al (2005), varios estudiantes en práctica inicial critican la falta de disponibilidad o interés de éste en desempeñar su rol, asumiendo esta función muchas veces por imposición. Lo mismo concluye Labra (2011), pues los estudiantes consideran escasas las oportunidades en que han tenido la compañía de un profesor guía reflexivo y comprometido, con interés en recibirlos. Según el estudio de OEI (2010), los estudiantes en práctica perciben que su quehacer no es legitimado, sintiéndose desvalorizados, aunque sus apreciaciones no coinciden con las de los docentes y Jefes de UTP entrevistados. Por su parte, los profesores supervisores, entrevistados en el estudio de Romero y Maturana (2012), consideran que los profesores guía entregan espacios de trabajo pero no retroalimentan el proceso de aprendizaje de los profesores en formación.

El saber profesional del docente guía es considerado por los futuros docentes como un referente importante en su formación. Sin embargo, en varios estudios los estudiantes en práctica coinciden en su apreciación de éste como un "antimodelo", produciendo en ellos un conflicto identitario con la profesión docente (Montecinos, 2010, Montecinos et al., 2012; OEI, 2010; Romero y Maturana; 2012; Solís et al., 2011). El estudio de Solís et al (2011) señala que sólo la mitad de las actividades observadas por los estudiantes en práctica, tanto en básica como en media, demuestran altos niveles de pensamiento y desafío intelectual para sus estudiantes; o bien, sólo la mitad de los practicantes observan actividades indagatorias conducidas con rigor académico. Preocupa esta situación a los investigadores por cuanto cuestiona tanto el tipo de actividades de aprendizaje como los estilos de enseñanza que aprenden los estudiantes en práctica. En consonancia con lo anterior, entre los aspectos más críticos percibidos por los futuros docentes con relación a la labor del profesor guía se mencionan: las precarias orientaciones de éste para establecer estrategias significativas de aprendizaje, para promover el pensamiento de sus estudiantes, para realizar adecuaciones curriculares, y para organizar los contenidos y objetivos de enseñanza (Romero y Maturana, 2012).

Con relación a las expectativas de los futuros docentes respecto de sus profesores guía, esperan de éstos asesoría técnico-pedagógica y que les ayuden a contextualizar la enseñanza (OEI, 2010). También demandan contención y apoyo emocional para su inserción e intervención exitosa en el centro educativo y con sus estudiantes (Hass, 2012; OEI 2010); 
y que éste actúe como un interlocutor reflexivo respecto de su desempeño (Montecinos et al., 2010; OEI, 2010). Sin embargo, uno de los aspectos más débilmente evaluados por los futuros profesores tanto en media como en básica, refiere a la retroalimentación y preguntas críticas con que guían su formación (Montecinos et al., 2010). Para este equipo de investigadores, el profesor o profesora guía debiera conocer las expectativas de aprendizaje, las tareas definidas para la asignatura de práctica, ofrecerle retroalimentación y ayudarlo a incorporarse a diversas actividades en el Centro. En síntesis, un aspecto de consenso en los estudios, con relación al rol del profesor guía, es la necesidad de mayor conciencia, orientación y diálogo con las instituciones formadoras de docentes y preparación de éstos para ejercer su función formativa.

\subsection{NECESIDAD DE PROGRESIVIDADY AUTONOMÍA EN LAS OPORTUNIDADES DE EJERCICIO DE LA DOCENCIA}

El tiempo dedicado a la enseñanza es limitado (44\% en básica y $66 \%$ en media), según Montecinos et al. (2010). Las tareas que desempeñan se pueden agrupar en tres tipos: colaboración al profesor, ayudantía a alumnos y docencia. Las oportunidades de ayudantía y docencia aumentan en el modelo concurrente y el consecutivo durante la práctica final. Núñez et al. (2011), concluyen que tanto en el modelo consecutivo como el concurrente se dan oportunidades variadas vinculadas a la preparación de la docencia, conducción de situaciones de enseñanza aprendizaje y evaluación de aprendizaje.

El grado de autonomía dado a los estudiantes es también un tema en cuestión. Los dos estudios anteriores confirman una progresión en los grados de autonomía, desde la práctica inicial a la final, aunque nunca se logra una autonomía total que permita a los futuros docentes decidir por sí mismos, sin autorización del profesor guía, el programa de docencia (Montecinos et al., 2010; Núñez et al., 2011).

Para los estudiantes en formación, es fundamental que los profesores guías les permitan libertad de acción en la sala de clases, acompañada posteriormente por una retroalimentación que les sirva para confrontar su quehacer y recibir sugerencias (OEI, 2010). Según este estudio, el tipo de relación que se da entre el profesor guía y el practicante puede llegar a oscilar entre dos extremos que generan tensión en los futuros docentes: una relación sobredelegadora, que exige una autonomía y carga de trabajo propia de un profesional, o una relación sobreestructurante con un alto nivel de imposición y control respecto de lo que éste debe hacer. La progresividad en los niveles de autonomía y el acompañamiento reflexivodialogante condicionan de manera clave las posibilidades de aprendizaje de la docencia.

Un aspecto no abordado en los estudios concierne a la figura de los estudiantes de escuelas y liceos en la experiencia de práctica. Este actor juega un rol importante en los aprendizajes de futuros profesores acerca de la docencia. Su agencia, sin embargo, no ha sido considerada como parte del proceso formativo en la construcción del saber pedagógico.

\subsection{UNA RELACIÓN DESARTICULADA ENTRE LAS CARRERAS DE PEDAGOGÍA Y LOS CENTROS DE PRÁCTICA}

El trabajo entre las carreras de pedagogía y las escuelas o centros de práctica se percibe desarticulado en la mayoría de los estudios. De manera general, se evidencia la falta de enlace entre el programa de práctica, la malla curricular de las carreras y las demandas 
que se plantean en la escuela (Labra, 2011; Montecinos et al., 2010; Nocetti et al., 2005; OEI, 2010). En su propuesta de lineamientos, Montecinos et al. (2010) recomiendan que la articulación de las carreras de pedagogía y las escuelas, a través del eje de prácticas, se convierta en una instancia que fortalezca la capacidad para que ambas instituciones ofrezcan en conjunto una educación de calidad. Hacer esto supone un trabajo conjunto para el diseño, implementación y evaluación del currículo de formación práctica, levantando "metas compartidas, combinando recursos de cada uno y generando normas y valores que provienen de los distintos contextos institucionales" (Montecinos et al., 2010: 238).

Los estudios revelan un contacto lejano y esporádico de las instituciones de formación inicial con las escuelas, lo que estaría afectando las facilidades de inserción de los futuros docentes (Labra, 2011; OEI, 2010). A nivel de la triada formativa, la desarticulación se refleja en el choque de expectativas y presiones de una y otra parte (OEI, 2010) y en los desacuerdos acerca del saber de la enseñanza, mencionado anteriormente (Gaete, 2003; Pardo, 2012; Romero y Maturana, 2012). Tanto los futuros docentes como los profesores guías plantean la necesidad de una mayor interrelación entre la universidad y la escuela. Para Labra (2011), parte de la explicación acerca de esta desarticulación se debe a que no se reconoce que los sujetos implicados en el proceso de formación son sujetos socio históricos cuyas percepciones y expectativas dialogan con los roles que les son asignados. Genera una enorme tensión en los miembros de la triada, la falta de una estructura de práctica orientada a explicitar estas creencias, expectativas, percepciones y representaciones. En los casos de estudio investigados, sólo en uno se intenciona una instancia de intercambio reflexivo entre miembros de la universidad y la escuela, al momento de evaluación de la experiencia de formación práctica, lo que permite contrastar esta diversidad de visiones y experiencias (Nocetti et al., 2005).

En esta misma línea, otra de las tensiones identificadas es que los centros de práctica solicitan al practicante realizar tareas que difieren de los requerimientos de la carrera (Labra, 2011; Montecinos et al., 2010; Nocetti et al.; 2005; OEI, 2010), sobre todo por la falta de orientación por parte de la universidad en el ejercicio de su labor, según revelan los profesores guía (Montecinos, 2010; Nocetti et al., 2005; OEI, 2010).

Un estudio reciente señala que los directivos de las instituciones educativas esperan que los estudiantes en práctica hagan un aporte en innovación educativa (Montecinos y Walker, 2011) o bien en actualización teórico-metodológica al aula (OEI, 2010). Esta expectativa también resulta problemática, ya que coloca al profesor guía en calidad de aprendiz y no al revés. De otra parte, muchas veces los futuros docentes aún no han adquirido los contenidos y competencias pedagógicas que se les exigen en la escuela, lo que genera su desmotivación y tensión en su desempeño en las escuelas (Nocetti et al., 2005). Para estos autores resulta evidente que las prácticas son valoradas cuando implican un trabajo orientado a un fin pedagógico bien definido y compartido por ambas partes; de lo contrario, las escuelas tienen una percepción negativa de esta experiencia y ofrecen resistencia a recibir estudiantes en práctica pues no tienen claro qué harán en la escuela. Rodrigo Fuentealba, en alusión a los planteamientos de Tom Russel, más que hablar de la generación de un "tercer espacio" al que se refieren los teóricos estadounidenses e ingleses, sugiere reconstruir la relación de la triada formativa. En ésta, tanto profesor supervisor y el profesor guía se definen y actúan como profesores colaboradores, lo que supone el cambio en la dinámica de poder entre éstos. De esta manera, tanto el docente universitario como el de la escuela son profesores en formación que requieren observar reflexivamente sus propias prácticas. Ambos están formando y formándose (comunicación personal, abril 2013). 
En definitiva, el desafío propuesto es "articular las visiones y experiencias de cada uno en un trabajo conjunto que tenga como foco el aprendizaje de los estudiantes del sistema escolar, de los estudiantes de pedagogía, de los profesores de aula y docentes de la universidad que acompañarán a los practicantes en este proceso formativo" (Montecinos et al., 2010).

\subsection{DIFICULTAD DE LOS FUTUROS DOCENTES PARA ACTUAR COMO "PROFESIONALES ADAPTATIVOS"Y DESARROLLAR RAZONAMIENTO PEDAGÓGICO}

Los estudios coinciden en cuanto al peso y valoración que adquiere la formación práctica dentro del currículo de formación inicial (Bobadilla et al., 2009; Labra, 2011; Nocetti et al., 2005; Walker, 2010). Los futuros docentes consideran que favorece el desarrollo de dos competencias principales: las pedagógicas y disciplinares. También, que las experiencias tempranas en los centros educativos fortalecen la vocación docente, la motivación por la carrera y el compromiso con su propia formación profesional (Nocetti et al., 2005). Asimismo, aumenta el porcentaje de estudiantes que declara sentirse bien o muy bien preparado para implementar procesos de enseñanza, en temas pedagógicos y didácticos (Montecinos, 2010).

Los tópicos cubiertos en los currículos de formación práctica de las 21 carreras estudiadas (Contreras et al., 2010; Montecinos, 2010) se clasificaron en los siguientes tipos: procesos e interacciones en el aula, actores, contexto escolar, investigación, contrastación teoría - práctica. Se observan algunas diferencias en la etapa en que se abordan los temas entre las carreras de básica y media. En ambas carreras, los resultados del estudio muestran que se invisibiliza o se relega a un segundo plano el desarrollar conocimiento respecto a los alumnos y a cómo ellos aprenden. De hecho, temas como aprendizaje y motivación de los estudiantes prácticamente están ausentes en los currículos analizados (Montecinos, 2010, Contreras et al, 2010). Esto contrasta con el mayor interés de los futuros profesores, que es conocer a los estudiantes y aprender a establecer relaciones interpersonales que posibiliten el aprendizaje en el aula (Montecinos, 2010; Contreras et al., 2010).

La definición acerca de qué se entiende por "saber docente" y cuál es el saber docente que se propone construir a través de la formación práctica, constituye un tema escasamente analizado. De acuerdo con las teorías que conciben el saber de la docencia como desarrollo de razonamiento pedagógico (Shulman, 1987), la conclusión de Labra (2011) es que la formación práctica no propicia procesos sistemáticos de apoyo al razonamiento sobre qué, cómo y con qué fin enseñar.

De acuerdo con la taxonomía de Shulman (1987), Labra resume los saberes identificados por los futuros docentes entrevistados en su estudio, en cuatro tipos: conocimiento del currículum, conocimiento pedagógico general, conocimiento de los estudiantes y conocimiento pedagógico del contenido. Según concluye Labra: los miembros de la triada formativa conciben la práctica final como de consolidación de conocimientos adquiridos (conocimientos teóricos) a través de su aplicación. El tipo de conocimiento profesional construido, se enmarca esencialmente con el saber hacer ${ }^{1}$. Es un conocimiento de tipo práctico y de rutinas de acción. Esta investigadora lo compara con "el saber práctico, de tipo personal y de proceso, saberes de la propia experiencia en la profesión, en el aula y en la escuela" (Labra, 2011: 166).

Construcción de estrategias para apoyar el trabajo de los estudiantes; elaboración de diseños de clases articulados con los objetivos planteados y con adecuada utilización de los tiempos; comprensión del funcionamiento/organización de la institución educativa; manejo/aplicación de técnicas que promueven el control de disciplina en la sala de clase; toma de decisiones adecuadas a la complejidad del contexto escolar; trabajo en equipo con colegas; ejecución de rutinas; valoración del conocimiento de los estudiantes; conocimiento y confianza en sí mismos (Labra, 2011: 166). 
Si bien los programas de Práctica Profesional analizados plantean como desafío el logro de la reflexión acerca de la práctica, los procesos de análisis y reflexión que los practicantes realizan han sido desarrollados por actitudes individuales y no por un acompañamiento sistemático y estructurado desde las instituciones universitarias. En sus hallazgos, Labra afirma que el nivel de reflexión alcanzado es básico, es decir, los estudiantes en práctica emplean estrategias elaboradas basadas en un conocimiento técnico y se analizan los resultados a partir de las reacciones de los estudiantes (Van Mannen, 1991; Leithwood, 1990 cit. por Labra, 2011). En este sentido, al no existir un apoyo sistemático y constante, no todos los practicantes lograron construir el mismo tipo de conocimiento profesional docente práctico.

Una vez enfrentados a la docencia, las responsabilidades profesionales para las cuales los futuros docentes declaran sentirse menos preparados son el trabajo con estudiantes con NEE y el trabajo con apoderados (Montecinos, 2010). Además, la evaluación aparece como un aprendizaje de la docencia con menor nivel de logro (Del Río, Lagos y Walker, 2011; Montecinos, 2010; Romero y Maturana, 2012). Se concluye que la formación práctica no estaría abordando el amplio rango de responsabilidades profesionales de la formación docente (Montecinos, 2010). A su vez, estos mismos estudios coinciden en la dificultad percibida por los futuros docentes para responder como "profesionales adaptativos", es decir, para contextualizar la enseñanza, especialmente en contextos de vulnerabilidad socio-educativa. Por último, se manifiesta como un problema el énfasis de los programas en las estrategias de enseñanza, sin que éstas sean analizadas de acuerdo con su impacto en los aprendizajes de los estudiantes (Del Río, Lagos y Walker, 2011).

El sentido de autoeficacia desarrollado por los futuros docentes con relación a dimensiones de su aprendizaje constituye objeto de estudio para Del Río, Lagos y Walker (2011). La fluctuación en la valoración de la autoeficacia en el desempeño pedagógico se mostró dependiente de diversos factores a lo largo de la formación práctica. Se evidencia que la dimensión en que los futuros docentes se sienten menos competentes es el manejo de la clase e interacciones en el aula, en comparación con su eficacia en el involucramiento de los estudiantes y eficacia en las estrategias de enseñanza. Los factores que marcan esa fluctuación dependen de aspectos como los enfoques en práctica, las condiciones de práctica, la etapa de práctica en la que se encuentran, el contexto escolar, etc.

Finalmente, existe un cuestionamiento al peso que tiene la formación inicial en el ejercicio de la docencia, sin embargo las conclusiones no son coincidentes. Mientras el estudio de Walker (2010) concluye que los currículos de formación docente llegan a tener más peso sobre las ideas de los futuros docentes acerca de la enseñanza y el aprendizaje; Labra (2011) halla que las indicaciones y aportes que pudiesen realizar aquellos que acompañan el proceso de práctica intermedia no estarían actuando como catalizadores de nuevas formas de actuación en los espacios de práctica profesional. En este sentido se plantea la necesidad de reconceptualizar el vínculo entre universidad y escuela, ya que no es posible construir el saber de la experiencia sin la experiencia de enseñar.

\section{LUCES ENTREGADAS POR LOS ESTUDIOS}

Los estudios realizados en Chile han puesto de manifiesto la gravitancia de la experiencia práctica en la formación del futuro docente, en tanto núcleo dinamizador del diálogo constructivo entre el saber de la teoría y el saber práctico. No sólo eso, el conocimiento 
profesional que se demanda de un docente es eminentemente práctico, y por tanto, es en este espacio donde el estudiante habrá de manifestar, deconstruir y reconstruir su saber pedagógico.

El predominio de un enfoque instrumental aplicacionista en las carreras de pedagogía, se explica no sólo por las concepciones imperantes acerca del saber de la enseñanza, sino también por los lineamientos que marcan las políticas educativas. Un lineamiento claro en este sentido lo marca la prueba INICIA, en la que se evalúa la calidad de la formación de un egresado de acuerdo con el nivel de conocimientos sobre la disciplina. En este sentido, resulta preocupante el carácter desprofesionalizante de la docencia expresado en la Ley General de Educación LEGE ( $\mathrm{N}^{\circ} 20.370$ ), definiendo que para la enseñanza de nivel medio no es necesario un título de profesor (Art. 46). Así es que educar se limita a instruir.

Como se ve en los estudios, el desarrollo de habilidades reflexivas como herramientas para la construcción del saber profesional docente supone adentrarse en la conceptualización y caracterización de la idea de "reflexión pedagógica" y cómo ésta se puede inducir y dinamizar para propiciar la actuación como "profesionales adaptativos". Para lograrlo se requiere un trabajo de acompañamiento sistemático y consistente por parte del supervisor y el profesor guía, junto con la conformación de comunidades de aprendizaje docente activas en su ejercicio reflexivo respecto de la práctica, tanto en las aulas de formación inicial como en las dinámicas profesionales de las escuelas. Todo parece apuntar a que poco de esto ocurre en ambos escenarios ${ }^{2}$. No existen estudios respecto al rol que cumplen otros actores en los procesos de reflexión pedagógica, tales como los compañeros de práctica, los coordinadores de práctica y otros docentes de la carrera, esto refleja la prevalencia de un enfoque individual del proceso reflexivo. Tampoco existen estudios acerca de la figura de los estudiantes de escuelas y liceos, considerando el importante rol que éstos juegan en los aprendizajes de la docencia. Al parecer, ésta es una expresión del énfasis de la práctica en la enseñanza más que de la relación entre enseñanza y aprendizaje y la escasa visibilidad de los escolares como actores relevantes en la construcción de la docencia.

Asimismo, ello supone clarificar cuál es el "saber docente" que se pretende movilizar, y en consecuencia, propiciar procesos sistemáticos de desarrollo de la capacidad de razonamiento pedagógico. Desde el punto de vista curricular, esto significa para las instituciones formadoras, articular e integrar los componentes del resto de la malla con los requerimientos del currículum de prácticas, consolidando una formación inicial congruente con este propósito, desde las diversas especialidades del currículo. A su vez, es evidente que las oportunidades de desempeño práctico requieren definirse más coherentemente en cuanto a su finalidad y pedagogía. No basta con aumentar el número de experiencias de práctica ni con desarrollar herramientas para registrar y evaluar, si las prácticas de enseñanza y los procesos de construcción del saber pedagógico no se ponen bajo análisis. Coincidentemente, éste es un ámbito de estudio que ha sido muy poco investigado en Chile. Como tampoco hay estudios sobre cómo se está evaluando la experiencia práctica.

La calidad del proceso de acompañamiento y mediación dada por el profesor supervisor constituye un factor clave para el desarrollo de razonamiento pedagógico. Este actor debiera adquirir mayor presencia, generándose condiciones de contratación que permitan un acompañamiento más sistemático y regular a los estudiantes en práctica. Además, su

Como bien señala el investigador Enrique Correa Molina (2014), el proceso de práctica permite movilizar y renovar las competencias profesionales no sólo del practicante, sino de los profesores que acompañan este proceso, reconociendo al mismo tiempo que la construcción de competencias no puede abstraerse del contexto. 
experticia debiera ser propiciada y fortalecida por la propia institución formadora. El escaso desarrollo de la capacidad de razonamiento pedagógico por parte de los estudiantes de pedagogía es congruente, a su vez, con la falta de reflexividad sobre su quehacer formativo como comunidad entre los formadores de formadores. En este sentido, se evidencia la necesidad de desarrollar políticas de formación de formadores en las carreras de pedagogía. Esto mismo se constata en el estado del arte sobre formación inicial en Chile (CEPPE, 2013) identificando un segundo grupo de falencias asociadas la falta de políticas para el desarrollo y perfeccionamiento del cuerpo académico de la institución formadora.

Un aspecto a resaltar es la falta de articulación entre las carreras y los centros de práctica. Esta desarticulación es el reflejo de la primacía del saber experto que se atribuye a la universidad. Como consecuencia, se desaprovecha el saber de la experiencia o la práctica en la academia y la posibilidad de renovación de la docencia escolar desde el saber proposicional (teoría). En este sentido, se requiere repensar la idea de profesionalización docente como un proceso continuo, que articula y retroalimenta la docencia universitaria, las experiencias de práctica, la inserción profesional de profesores principiantes y dentro de ello la labor clave de los profesores guías. El rol indefinido y solitario del profesor guía dentro de la institución escolar evidenciado en los estudios es una muestra de la invisibilidad de la importante labor formativa que éstos pueden desempeñar en los procesos de construcción del saber pedagógico desde la práctica. Dentro de la carrera docente se recomienda relevar la función y estatus de determinados perfiles profesionales, como el de los profesores guía o profesores mentores, ofreciéndoles una formación que potencie sus competencias y que les entregue herramientas específicas para que cumplan dicha función. Además se propone considerar sus condiciones de desempeño, como la reducción de su carga académica dentro de las instituciones escolares, para que éstos aporten su conocimiento pedagógico y experiencia y combinen la docencia y acompañamiento con su participación en los equipos profesionales de práctica de las instituciones de formación docente. A su vez, se requiere fortalecer el vínculo con los centros escolares, estableciendo una red de escuelas con las que se articula y trabaja de manera preferente la institución formadora de docentes. En este sentido, resulta urgente la regulación de la oferta de formación inicial que permita ordenar y articular con criterio de calidad las prácticas en las escuelas. La excesiva demanda de prácticas, libradas muchas veces a la búsqueda de los propios estudiantes de pedagogía, se ha transformado en una enorme circulación de estudiantes de pedagogía de distintos planteles por una misma institución escolar, sin que exista un programa de inserción regulado al interior de éstas.

Desde la perspectiva de los marcos políticos orientadores de la formación inicial docente, surgen diversas preguntas ¿En qué medida instrumentos de mejora, como los actuales Convenios de Desempeño docente se inscriben en un enfoque crítico reflexivo? ¿Qué ideas acerca del saber de la enseñanza maneja el MECESUP para la aprobación, seguimiento y evaluación de los Convenios? ¿En qué medida este instrumento propicia e incentiva la reflexión pedagógica a nivel de las carreras y contribuye así a instalar procesos continuos de reflexión y mejora? En las bases de los Convenios de Desempeño, por cierto, instrumento competitivo para la adjudicación de recursos en base a resultados, se establece como uno de sus cinco componentes la renovación y fortificación del cuerpo académico, lo que implica establecer una política institucional de perfeccionamiento académico. Según informa el estudio CEPPE 2013, el componente de formación práctica ha cobrado relevancia en estos convenios y apuntan a una red de cooperación con los establecimientos 
educacionales y un programa de capacitación para profesores guía. Además, algunas instituciones proponen un rediseño que profundice el carácter reflexivo y de investigación de la formación docente. Si la aspiración política es asegurar la calidad de la educación superior en las carreras de pedagogía, estos convenios debieran regir para todas las carreras de pedagogía en Chile para que estas sean acreditadas.

La misma pregunta surge respecto del Sistema de Acreditación de las carreras de pedagogía ¿Qué parámetros utiliza y cómo los aplica? ¿Qué concepciones acerca del saber de la enseñanza manejan las instituciones acreditadoras? Una de las falencias que presenta el sistema de acreditación es la falta de criterios de acreditación propios de las carreras de pedagogía (CEPPE, 2013). Entre otros estudios, citan el informe de la OCDE (2009) que señala que los criterios de acreditación no se orientan a medir estándares de procesos, condiciones institucionales y resultados.

Tal como propuso la Comisión sobre Formación Inicial Docente convocada el 2005 por el MINEDUC, para subsanar las faltas y debilidades en las que incurren las instituciones de formación inicial de docentes se insiste en la necesidad de articular un trabajo conjunto entre instancias de definición política como son el Ministerio de Educación (Centro de Perfeccionamiento e Investigaciones Pedagógicas [CPEIP] incluido), sostenedores, el Sistema de Acreditación, División de Educación Superior (Programa MECESUP) y el Colegio de Profesores; y en lo académico las facultades de educación, los centros de estudios, cuerpo de profesores organizados en distintos movimientos. De modo que cualquier iniciativa que se tome en instancias políticas dialogue estrechamente con lo que sucede con las instancias más académicas y de ejecución, para la articulación y coherencia sistémica de la formación inicial, la formación continua y la carrera docente. Para ello es indudable la necesidad de una institucionalidad que entienda la complejidad de la profesión docente y que oriente, apoye y monitoree los procesos de profesionalización docente.

\section{REFERENCIAS BIBLIOGRÁFICAS}

Abarca, V., Castaño J. y Barahona C. (2003). Evaluación de las competencias profesionales desarrolladas durante el proceso de formación de las prácticas tempranas y práctica profesional de los alumnos de la carrera de Educación Básica y Educación Media en la Universidad de Playa Ancha. Presentación en Congreso ENIN 2003. Universidad de Playa Ancha de Ciencias de la Educación, Valparaíso

Ávalos, B. (2002). Profesores para Chile, Historia de un proyecto. Santiago de Chile: Ministerio de Educación.

Ávalos, B. y Matus, C. (2010). La Formación Inicial Docente en Chile desde una óptica Internacional. Informe Nacional del Estudio Internacional IEA TEDS-M. Santiago de Chile: Ministerio de Educación.

Bobadilla, M., Cárdenas, A., Dobbs, E. y Soto, A. (2009). Los rodeos de la práctica. Representaciones sobre el saber docente en el discurso de estudiantes de pedagogía. Estudios Pedagógicos, vol.35, n.1, 239-252.

CEPPE. (2013). La Formación Inicial Docente: Estado, Avances y Desafíos en Chile. Informe Final Servicio de Asesoría sobre FID. Santiago de Chile: Autor.

Cisternas, T. (2011). La investigación sobre formación docente en Chile. Territorios explorados e inexplorados. Calidad en la educación, n.35, 131-164.

Comisión sobre Formación Inicial Docente. (2005). Informe Comisión sobre Formación Inicial 
Docente. Documento no oficial del Ministerio de Educación.

Contreras, I., Rittershaussen, S., Montecinos, C., Solís, M. C., Núñez, C. y Walker, H. (2010). La escuela como espacio para aprender a enseñar: visiones desde los programas de formación de profesores de educación media. Estudios Pedagógicos, vol.36, n.1, 85-105.

Correa, E. (2014). Las prácticas: primer espacio de profesionalización docente. En C. Hirmas y I. Cortés (Eds.), Primer Seminario sobre Formación Práctica Docente: Vinculación entre el sistema universitario y el sistema escolar (pp. 25-40). Valparaíso: Organización de Estados Iberoamericanos para la Educación de la Ciencia y la Cultura.

Del Río, M., Lagos, C. y Walker, H. (2011). El efecto de las experiencias de práctica en el desarrollo del sentido de autoeficacia en la formación inicial de educadoras de párvulos. Estudios Pedagógicos, vol.37, n.1, 149-166.

Gaete, M. (2003). Evaluación de las prácticas profesionales: El desafío de evaluar procesos. Presentación en Congreso Internacional de la Función Docente, Universidad de La Frontera, Temuco.

Galaz, A. y Fuentealba, R. 2008. La reflexión como recurso para la mejora de las prácticas docentes en servicio: el caso de las Redes Pedagógicas Locales. En J. Cornejo y R. Fuentealba (Eds.), Prácticas reflexivas para la formación profesional docente ¿qué las hace eficaces? (pp. 141-168). Santiago: Ediciones UCSH.

Haas, V. (2012). Una aproximación a las características personales del buen mentor desde las concepciones de los propios estudiantes de práctica final de Educación Básica de la Pontificia Universidad Católica de Valparaíso. Presentación en III Congreso Internacional sobre Profesorado Principiante e Inserción Profesional a la Docencia, Universidad Autónoma de Chile, Santiago de Chile.

Inostroza de Celis, G. (1997). La práctica, motor de la formación docente. Santiago: Dolmen Ediciones.

Labra, P. (2011). Construcción de conocimiento profesional docente: el caso de la formación en la práctica (Tesis para optar al Grado Académico de Doctor en Educación). Universidad Academia de Humanismo Cristiano, Santiago.

Labra, P., Montenegro, G., Iturra, C. y Fuentealba, R. (2005). La investigación-acción como herramienta para lograr coherencia de acción en el proceso de práctica profesional durante la formación inicial docente. Estudios Pedagógicos, vol.31, n.2, 137-143.

Latorre, M. (2006). Nuevas miradas, viejos problemas: las relaciones entre formación inicial y ejercicio profesional docente. Foro Educacional, n.10, 41-64.

LEY 20.129. (2006). Sistema Nacional de Aseguramiento de la Calidad de la Educación Superior. Biblioteca del Congreso Nacional de Chile.

LEY 20.370. (2009). Ley General de Educación. Biblioteca del Congreso Nacional de Chile.

Marcelo, C. (1999). Formación del Profesorado para el cambio educativo. Barcelona: Ed. EUB.

Molina, P. (2008). Práctica docente progresiva en la formación inicial desde un enfoque reflexivo crítico. En R. Fuentealba y J. Cornejo (Eds.), Prácticas reflexivas para la formación profesional docente: ¿qué las hace eficaces? (pp. 13-27). Santiago de Chile: Ediciones UCSH.

Montecinos, C. (2010). El impacto de la formación práctica en el aprender a enseñar FONDECYT N ${ }^{\circ}$ 1070807. Santiago de Chile.

Montecinos, C., Walker, H., Solís, M., Núñez, C., Contreras, I. y Rittershaussen, S. (2010). Lineamientos para el Diseño del Currículo de Formación de Carreras de Pedagogía. En S. Martinic y G. Elaqua, ¿Fin de ciclo? Cambios en la gobernanza del sistema educativo (pp. 233-256). Santiago: ORALC/UNESCO. Pontifica Universidad Católica de Chile.

Montecinos, C., y Walker, H. (2011). La contribución de los centros de práctica a la formación inicial docente: Perspectivas de docentes de aula y docentes directivos. FONDECYT N ${ }^{\circ} 1110505$.

Montecinos, C., Barrios, C. y Tapia, M. F. (2011). Relación entre estilos de supervisión durante la práctica profesional y las creencias de autoeficacia de los estudiantes de Pedagogía en Educación Básica. Perspectiva Educacional, vol.50, n.2, 96-122. 
Montecinos, C., Walker, H., Cortez, M. y Maldonado, F. (2012). ¿Por qué y para qué los centros escolares reciben a estudiantes de pedagogía en práctica? Perspectivas de docentes directivos. Páginas de Educación, vol.6, n.1, 37-59.

Montenegro, H. y Fuentealba, R. (2012). Prácticas de enseñanza para la formación de futuros profesores: Propuesta de un modelo para su estudio. Presentación en III Congreso Internacional sobre Profesorado Principiante e Inserción Profesional a la Docencia Universidad Autónoma de Chile, Santiago de Chile.

Nocetti de la Barra, A., Mendoza, M., Contreras, G., Sanhueza, K. y Herrera, S. (2005). Caracterización de las prácticas iniciales de las carreras de pedagogía en universidades chilenas. Concepción: Universidad San Sebastián. FONIDE.

Núñez, C., Solís, M., Contreras, I., Vásquez, N. y Rittershaussen, S. (2011). Oportunidades de desempeño de los estudiantes de pedagogía media en la formación práctica. Contribución Seminario Internacional en Práctica Pedagógica De la teoría a la práctica, Universidad Francisco de Paula Santander, Cúcuta, Colombia.

Organización de Estados Iberoamericanos. (2010). Percepciones en torno al rol y las competencias del profesor guía por parte de actores educativos involucrados en la experiencia de práctica profesional docente en la región metropolitana. Santiago de Chile: OEI.

OCDE. Banco Internacional para la Reconstrucción y el Desarrollo. Banco Mundial. (2009). Revisión de Políticas Nacionales de Educación. La Educación Superior en Chile. Santiago, París: Autor, MINEDUC.

Ortúzar, S., Flores, C., Milesi, C. y Cox, C. (2009). Aspectos de la formación inicial docente y su influencia en el rendimiento académico de los alumnos. Santiago de Chile: Pontificia Universidad Católica de Chile.

Pardo, L. A. (2012). Desarrollo de la capacidad reflexiva docente en las experiencias laborales: el caso de la carrera de pedagogía en inglés. Presentación en III Congreso Internacional sobre Profesorado Principiante e Inserción Profesional a la Docencia, Grupo de Investigación Idea. Universidad Autónoma de Chile, Santiago

Ritterhaussen, S., Contreras, I., Suzuki, E., Solís, M.C. y Valverde, P. (2004). Prácticas Iniciales en la formación de educadores. Revista De Investigación Educacional Latinoamericana, vol.35, n.2, 173-191.

Romero, M. y Maturana, D. (2012). La supervisión de prácticas pedagógicas. Magis, Revista Internacional de Investigación en Educación, vol.4, n.9, 653-667.

Shulman, L. S. (1987). Knowledge and teaching: foundations of new reform. Harvard Educational Review, vol.57, n.1, 1-22.

Solís, M. C., Núñez, C., Contreras, I., Rittershaussen, S., Montecinos, C. y Walker, H. (2011). Condiciones de la formación práctica de los futuros profesores. Estudios Pedagógicos, vol.37, n.1, 127-147.

Walker, H. (2010). Aprendiendo a enseñar desde la perspectiva de estudiantes de pedagogía en educación media. FONDECYT N 1070807.

Zeichner, K. (2010). Nuevas epistemologías en formación del profesorado. Repensando las conexiones entre las asignaturas del campus y las experiencias de prácticas en la formación del profesorado en la universidad. Revista Interuniversitaria de Formación de Profesorado, vol.24, n.2, 123-149. 
